\title{
Molecular features assisting in diagnosis, surgery, and treatment decision making in low-grade gliomas
}

\author{
${ }^{*}$ Ricky Chen, MD, ${ }^{1}$ Vijay M. Ravindra, MD, ${ }^{2}$ Adam L. Cohen, MD, ${ }^{3}$ Randy L. Jensen, MD, PhD, ${ }^{2}$ \\ Karen L. Salzman, MD, ${ }^{4}$ Andrew P. Prescot, PhD, ${ }^{5}$ and Howard Colman, MD, PhD ${ }^{2}$ \\ ${ }^{1}$ Department of Neurology, Clinical Neurosciences Center; ${ }^{2}$ Department of Neurosurgery, Huntsman Cancer Institute and Clinical \\ Neuroscience Center; ${ }^{3}$ Division of Oncology, Huntsman Cancer Institute; ${ }^{4}$ Department of Radiology, Division of Neuroradiology; \\ and ${ }^{5}$ Department of Radiology and Brain Institute, University of Utah, Salt Lake City, Utah
}

\begin{abstract}
The preferred management of suspected low-grade gliomas (LGGs) has been disputed, and the implications of molecular changes for medical and surgical management of LGGs are important to consider. Current strategies that make use of molecular markers and imaging techniques and therapeutic considerations offer additional options for management of LGGs. Mutations in the isocitrate dehydrogenase 1 and 2 (IDH1 and IDH2) genes suggest a role for this abnormal metabolic pathway in the pathogenesis and progression of these primary brain tumors. Use of magnetic resonance spectroscopy can provide preoperative detection of IDH-mutated gliomas and affect surgical planning. In addition, IDH1 and IDH2 mutation status may have an effect on surgical resectability of gliomas. The IDH-mutated tumors exhibit better prognosis throughout every grade of glioma, and mutation may be an early genetic event, preceding lineage-specific secondary and tertiary alterations that transform LGGs into secondary glioblastomas. The O6-methylguanine-DNAmethyltransferase (MGMT) promoter methylation and $1 p 19 q$ codeletion status can predict sensitivity to chemotherapy and radiation in low- and intermediate-grade gliomas. Thus, these recent advances, which have led to a better understanding of how molecular, genetic, and epigenetic alterations influence the pathogenicity of the different histological grades of gliomas, can lead to better prognostication and may lead to specific targeted surgical interventions and medical therapies.
\end{abstract}

http://thejns.org/doi/abs/10.3171/2015.1.FOCUS14745

KEY WORDS glioma; brain tumor; isocitrate dehydrogenase; oligodendroglioma; astrocytoma; magnetic resonance spectrometry; D-2-hydroxyglutarate spectrometry

$\mathrm{T}$ HE ideal management of suspected low-grade gliomas (LGGs) has historically been controversial in neurosurgery and neurooncology. ${ }^{20,22,38}$ The majority of low-grade glial tumors are characterized by diffuse brain infiltration and are not surgically curable. ${ }^{7}$ Although patients diagnosed with infiltrating LGGs (Grade II) live much longer than patients with high-grade gliomas (Grades III and IV), there is substantial heterogeneity in outcome, even in patients who share the same initial diagnosis..$^{14}$ Although previous reports had shown that expectant management is safe until there is clear disease progres- sion, ${ }^{28,29}$ most recent and large series have reported longer survival and time to malignant transformation if total resection of the tumor is achieved. $1,8,25,30,31$

The identification of mutations in the isocitrate dehydrogenase 1 and 2 (IDHI and IDH2) genes in WHO Grades II, III, and IV gliomas has directed attention to the role of this abnormal metabolic pathway in the pathogenesis and progression of these primary brain tumors., ${ }^{3,40}$ Unlike IDH3, which participates in the Krebs cycle, IDH1 and IDH2 are cytosolic; thus the mutations are confined to the active site and result in a change of function that generates D-

ABBREVIATIONS EORTC = European Organisation for Research and Treatment of Cancer; G-CIMP = glioma CpG island methylator phenotype; GBM = glioblastoma multiforme; HR = hazard ratio; IDH = isocitrate dehydrogenase; LGG = low-grade glioma; MGMT = O6-methylguanine-DNA-methyltransferase; MRS = MR spectroscopy; OS = overall survival; PCV = procarbazine, lomustine, and vincristine; PFS = progression-free survival; RTOG = Radiation Therapy Oncology Group; ${ }^{1} \mathrm{H}$ HR-MAS = proton high-resolution magic angle spinning; $2 \mathrm{HG}=\mathrm{D}$-2-hydroxyglutarate.

SUBMITTED October 31, 2014. ACCEPTED January 7,2015

INCLUDE WHEN CITING DOI: 10.3171/2015.1.FOCUS14745.

DISCLOSURE Dr. Jensen is a consultant for Varian, Pharmaco-Kinesis, Medtronic, and IMRIS. Dr. Colman is a consultant for Hoffman La Roche, Genentech, Sigma Tau, Proximagen, and Foundation Medicine.

* Drs. Chen and Ravindra contributed equally to this work. 
2-hydroxyglutarate (2HG) rather than the normal products of NADPH (nicotinamide adenine dinucleotide phosphate [reduced form]) and $\alpha$-ketoglutarate. ${ }^{12}$ It is thought that 2HG alters cellular genetic and epigenetic programs, such as histone demethylation, hypoxia sensing, and induction of DNA hypermethylation, leading to tumorigenesis. . $^{5,10,16,35}$ Methylation of the DNA repair protein O6-methylguanineDNA-methyltransferase (MGMT) gene promoter is correlated with IDH mutation, and this may perhaps be attributed to the global DNA hypermethylation effects of the $2 \mathrm{HG}$ oncometabolite. ${ }^{24}$

A better understanding of these and other molecular changes in gliomas and their oncometabolic sequelae has changed the way in which various histological grades of gliomas are being diagnosed and treated. Laboratory testing of molecular features including $I D H$ status, $1 p 19 q$ codeletion, and $M G M T$ promoter methylation has offered new insights on prognosis and management, including predicted response to chemotherapy and radiation treatment. Recently, 2 separate groups have reported results on the successful noninvasive detection of $2 \mathrm{HG} .{ }^{9,14}$ Use of MR spectroscopy (MRS) can provide preoperative detection of $I D H$-mutated gliomas and affect surgical planning. In addition, the impact of $I D H I$ and $I D H 2$ mutation status on surgical resectability of gliomas has been explored recently. ${ }^{4}$ Previous reviews have addressed the evidence behind $I D H 1$ and $1 p 19 q$ codeletion as major prognostic features for gliomas. In this article, we review these key concepts and provide a general summary of the molecular implications for medical and surgical management of LGGs.

\section{Diagnostic and Prognostic Implications of IDH Mutation}

In contrast to glioblastoma multiforme (GBM), the majority (65\%-90\%) of LGGs, which include astrocytoma, oligodendroglioma, and oligoastrocytoma, harbor mutations in $I D H$, with most of these being $I D H I$ mutations. ${ }^{33}$ This strong association between LGGs and $I D H$ mutation has several diagnostic implications. The first is the plausible hypothesis that IDHI-mutant GBMs represent malignant transformation of undiagnosed LGGs, and IDHI wild-type GBM is, in many ways, a different disease sharing the same histological features. ${ }^{11,13,37}$ This theory is supported by studies of serial biopsy samples obtained in patients with glioma, in which the IDHI mutation appears to be a relatively early genetic event, ${ }^{11,13,37}$ preceding other lineage-specific mutations, such as TP53 and ATRX mutations in astrocytomas and $1 p 19 q$ codeletion, CIC , and telomerase reverse transcriptase (TERT) mutations in oligodendrogliomas. ${ }^{13,34,37}$

In The Cancer Genome Atlas studies, IDHI-mutant GBMs cluster by gene expression in the proneural subtype. ${ }^{5,11}$ The $I D H I$ mutation initiates a cellular program through the effects of the oncometabolite $2 \mathrm{HG}$, which appears to modulate cellular programs including hypoxia sensing, histone demethylation, and induction of a globally hypermethylated state of DNA and angiogenesis. ${ }^{13}$ This hypermethylated state of DNA, known as glioma $\mathrm{CpG}$ island methylator phenotype (G-CIMP), is prognostically favorable. ${ }^{5,11} \mathrm{By}$ gene expression, all GBMs with the
G-CIMP phenotype are proneural, but not all proneural GBMs are G-CIMP ${ }^{32}$ The $I D H$ mutation is thought to play a causal role in creation of the G-CIMP phenotype and may be responsible for much of its survival advantage. ${ }^{11}$ Thus, IDHI genetic mutation is thought to be an early and perhaps the initiating event leading to tumor development. Based on these insights, IDHI wild-type GBMs are now seen as primary GBMs that probably do not share the same pathophysiological features as secondary GBMs that arise from LGGs with $I D H$ mutation ${ }^{11}$ and associated subsequent secondary and tertiary mutations that cause their malignant transformation. ${ }^{37}$ Therefore, IDHI wild-type GBMs behave much differently than their IDHI-mutant counterparts, with a later age of onset, worse prognosis, and distinct imaging characteristics. . $^{13,34,35}$

A second diagnostic implication of the relationship between tumor grade and $I D H$ status is that LGGs that are $I D H$ wild type clinically tend to behave as aggressively as GBM. ${ }^{13}$ Among gliomas of the same histopathological grade, the presence of $I D H 1$ or $I D H 2$ mutation confers a significantly better prognosis. A meta-analysis of 937 patients showed that among patients with LGG, those whose lesions had $I D H$ mutation had a more favorable prognosis (pooled hazard ratio [HR] of $0.585, \mathrm{p}=0.025$ ) compared with $I D H$ wild-type LGGs. ${ }^{33}$ In the NOA-04 trial of 318 patients with anaplastic glioma, multivariate analyses also showed that IDHI mutation was an independent prognostic factor that conferred a longer progression-free survival (PFS) irrespective of the treatment received, and this effect was statistically greater than the risk reduction seen with $1 p 19 q$ codeletion, $M G M T$ promoter methylation, or histological features. ${ }^{39}$ Conversely, the absence of $I D H$ mutation in an intermediate- or even low-grade glioma portends a potentially worse prognosis than that of a GBM that has IDH mutation. ${ }^{33}$

Codeletion of $1 p 19 q$ and $M G M T$ promoter methylation are also independent markers of a favorable prognosis that appear to increase the sensitivity of tumors to radiation and chemotherapy. ${ }^{39}$ Silencing of the MGMT promoter by methylation has been shown in prior trials to benefit patients with GBM who receive treatment with temozolomide. ${ }^{17}$ Thus, the use of molecular markers provides important prognostic information that can guide decision making in the treatment of LGGs and intermediate-grade gliomas.

\section{Relationship of IDH, 1p19q, MGMT, and Other Markers}

In 2013, Leu et al. ${ }^{24}$ analyzed the interrelationship of molecular markers in LGG by assessing their correlation with each other and their independent and combined prognostic predictive value. They found that $M G M T$ promoter methylation and $I D H$ mutation are strongly correlated, with nearly all MGMT methylated tumors having $I D H$ mutation, but only about half of $M G M T$ unmethylated tumors having $I D H$ mutation. $1 p 19 q$ codeletion and $I D H$ mutation also have a strong correlation, and essentially all tumors with $1 \mathrm{p} 19 \mathrm{q}$ deletion also have $I D H$ mutation; however, the relationship between $I D H$ and $p 53$ is less correlated. In terms of survival associations in their study, $I D H$ 
mutation combined with $M G M T$ methylation had a favorable impact on overall survival (OS) compared with $I D H$ wild type (HR 0.33, p < 0.01). Further combination of $I D H$ mutation and MGMT methylation with $1 p 19 q$ codeletion (triple combination) resulted in an even greater survival benefit (HR 0.18, p < 0.001). The results of this study suggest not only that these molecular markers exhibit varying degrees of correlation and prognostic influence, but also that their effects can be independent and additive.

These specific combinations of alterations are also changing the current view of pathological diagnosis, and future WHO classifications are expected to include some of these markers. In addition, a number of novel alterations have been associated with specific histopathological-molecular subtypes. For example, most infiltrating astrocytomas and secondary GBMs have mutations in TP53 and $A T R X$ in addition to $I D H$. Oligodendrogliomas have $I D H$ mutation and $1 p 19 q$ codeletion, but also have high frequencies of mutations in CIC, FUBPI, and TERT promoter. In contrast, the vast majority of primary GBMs lack IDH mutations, but instead are characterized by mutations or copy number alterations in EGFR, PTEN, TP53, PDGFRA, $N F 1$, and $C D K N 2 A / B$ and the TERT promoter. ${ }^{2}$ Some of these markers also carry prognostic significance within subgroups. For example, 1 study identified PDGFRA amplification as an independent prognostic factor within IDHI-mutated GBMs that is associated with a worse prognosis. ${ }^{26}$

Work with orthotopic xenografts of IDHI-mutated gliomas has identified tertiary alterations, including the PIK3CA and KRAS mutations as well as PDGFRA, MET, and N-Myc amplification, which appear to be involved in the eventual malignant transformation of LGGs. ${ }^{37}$ Retrospective analysis of 149 patients with gliomas showed that tertiary alterations were present in $13.4 \%$ of the sample and were found exclusively in high-grade gliomas or progressive LGGs. The development of tertiary alterations after progression in LGGs was associated with significantly shorter subsequent PFS (median 9 vs 36.1 months, $\mathrm{p}=0.0011$ ), whereas there was no significant difference in PFS from the time of initial diagnosis. Again, these data support the fundamental tenet of cancer biology that "stepwise acquisition of distinct classes of mutations results in more aggressive disease." In the "molecular evolutionary tree of gliomas," IDH1/2 mutation represents an early "trunk mutation" that precedes the "lineage-defining" secondary alterations of TP53 mutation and ATRX mutation in astrocytomas and $1 p 19 q$ codeletion with $C I C$ and FUBP1 mutation in oligodendrogliomas, and "tertiary alteration detected at progression [is] the driver of malignant degeneration." These authors observed that the ability to form xenografts was enhanced by the presence of these tertiary alterations and that selective inhibitors of their downstream pathways were able to suppress proliferation in vitro, suggesting that these mutations are oncogenic drivers of transformation. ${ }^{37}$

\section{Novel Preoperative Molecular Testing and Imaging for Surgical Planning}

The strong link between LGGs and $I D H$ mutation can be exploited to improve the accuracy of tumor diagnosis, which traditionally has relied solely on histopathological analysis of resection. For example, in a prospective analysis comparing 246 patients with GBM and 157 with anaplastic astrocytomas, Kim et al. ${ }^{21}$ found that histopathological diagnosis of GBM was highly dependent on the volume of resection. Smaller resections that were $<20 \mathrm{ml}$ resulted in a much lower rate of diagnosis of GBM than was seen with larger resections $>20 \mathrm{ml}$. In contrast, $I D H 1$ mutation status did not correlate with the size of resection. ${ }^{21}$ This indicates a greater likelihood of underdiagnosis of GBM with smaller resections, and molecular genotyping for $I D H I$ mutation could be used to increase the diagnostic accuracy.

In 2012, Elkhaled et al. ${ }^{14}$ described a technique of ex vivo detection of $2 \mathrm{HG}$ in which the nuclear MR technique of proton high-resolution magic angle spinning ( ${ }^{1} \mathrm{H}$ HRMAS) spectroscopy was used for patients with recurrent LGGs. The investigators collected a total of 104 tissue samples from 52 patients; assessment of the $I D H 1$ status yielded 40 patients classified as positive for $I D H I$ mutations and 12 as IDHI negative. In addition, 26 of 31 patients whose lesions had converted to a higher grade at the time of recurrence were classified as IDHI mutated compared with 14 of the 21 whose lesions had remained Grade II. There was an $86.4 \%$ concordance rate between the presence of $2 \mathrm{HG}$, as detected by ${ }^{1} \mathrm{H}$ HR-MAS spectroscopy, and IDHI mutation status, as determined by antibody staining and genetic sequencing..$^{14}$

In 2012, Choi et al. ${ }^{9}$ similarly reported the noninvasive detection of $2 \mathrm{HG}$ by proton MRS. They used a method of spectral fitting to estimate the concentrations of $2 \mathrm{HG}$ in the tumors of 30 patients. Indeed, they correlated the detection of $2 \mathrm{HG}$ with mutations in IDHI and IDH2 and with increased levels of $2 \mathrm{HG}$ by mass spectrometry of resected tumor.

These 2 studies identified a direct metabolic consequence of a genetic mutation in a cancer cell through noninvasive imaging. ${ }^{9}, 14$ Although there are technical limitations to be considered prior to bringing these technologies to everyday practice, including signal overlaps with $\gamma$-aminobutyric acid, glutamate, and glutamine, and increased water concentration from edema, which could underestimate the concentrations of metabolites, these parameters may be adjusted for using MRS sequence optimization and through the use of a phantom, respectively. ${ }^{9}, 19$ The use of $2 \mathrm{HG}$ detection as an adjuvant in assessing the presence of $I D H 1$ and $I D H 2$ mutation when evaluating a brain lesion may have future implications for surgical planning. Using this novel imaging technique not only enables clinicians to distinguish tumor from normal brain or nonneoplastic process (such as demyelinating disease), but the presence of $2 \mathrm{HG}$ spectra in vivo may make possible presurgical detection of $I D H$ mutation in a suspected glioma (Fig. 1).

In 2012, Pope et al..$^{27}$ demonstrated in 27 patients that MRS detected elevated 2HG levels in gliomas with IDHI mutations compared with wild type $(\mathrm{p}=0.003)$ and, when measured in vivo, these levels were significantly correlated with those measured via MRS $(p=0.0001)$. These findings can provide essential preoperative information about $\mathrm{IDHI}$ status to neurosurgeons, which may impact the extent of resection that is desired. ${ }^{4}$ 

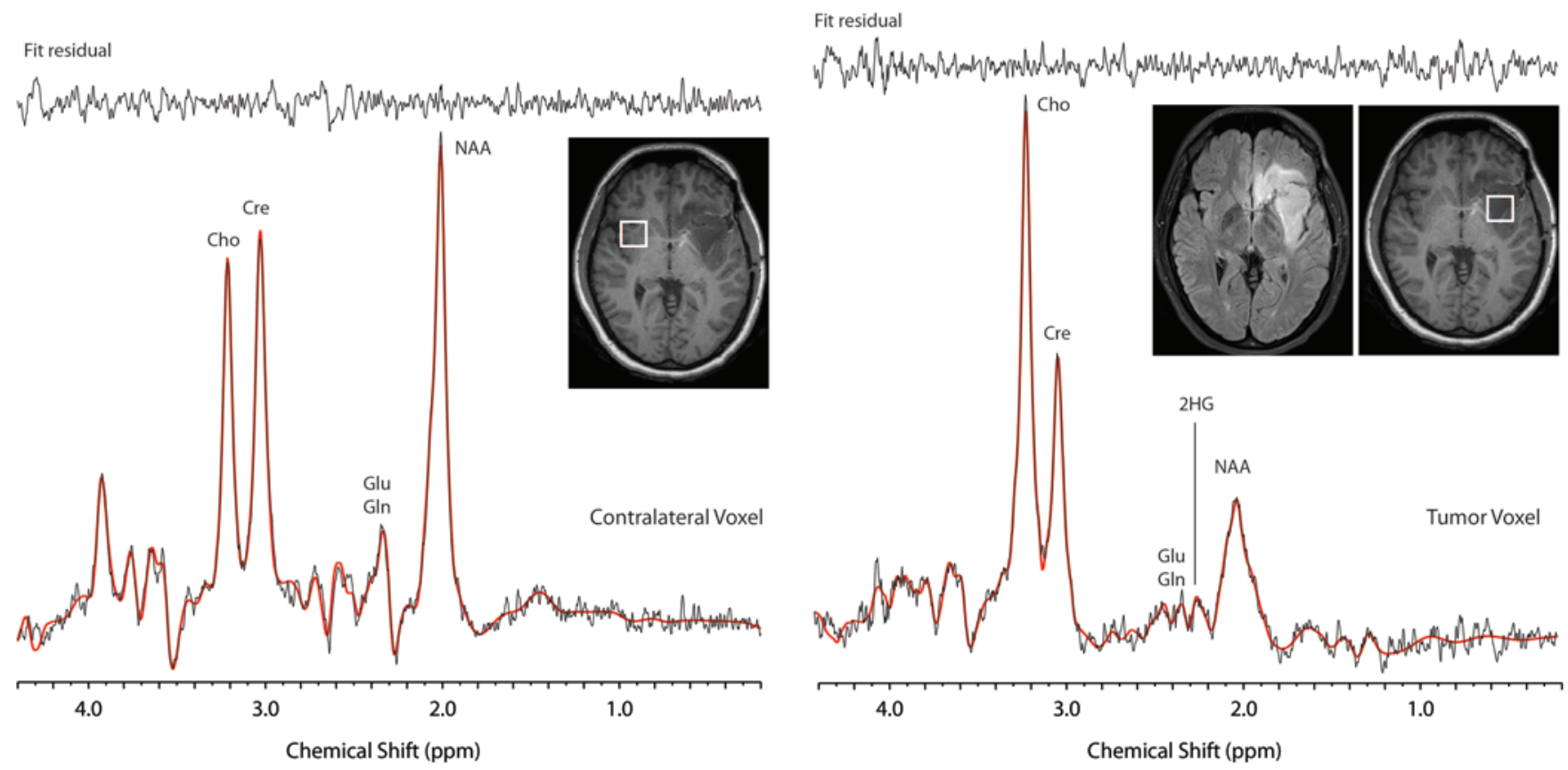

FIG. 1. In vivo ${ }^{1} \mathrm{H}$ MRS brain spectra from a patient presenting with an IDH-mutated glioma. All data were obtained from $2 \times 2 \times 2-$ $\mathrm{cm}^{3}$ voxels positioned within the lesion (right) as seen on the FLAIR and T1-weighted MR images (insets) and normal-appearing brain tissue contralateral to tumor location (left) as seen on the T1-weighted MR image (inset), by using a point-resolved spectroscopy sequence at 3 T with TE $97 \mathrm{msec}$, according to the technique developed by Choi et al. Both panels display the spectral fits (red line spectra) overlaid on the raw spectral data (black line spectra), and fit residuals (raw data minus fit) are presented above both spectral fits. Normal brain spectra show significant singlet resonances attributable to $N$-acetylaspartate (NAA) (2 ppm), creatine (Cre) (3 ppm), and choline (Cho) (3.2 ppm) methyl protons, and glutamic acid (Glu) and glutamine (GIn) form a spectral complex displaying a dispersive character between 2.2 and $2.45 \mathrm{ppm}$. In contrast, the ultimate fitting of ${ }^{1} \mathrm{H}$ MRS data recorded from the IDH-mutated glioma clearly identifies the significant presence of $2 \mathrm{HG}$ accompanied by elevated Cho concentration, reduced NAA, and lower levels of Glu and GIn.

\section{Surgical Planning and Resection}

Surgical intervention for LGGs is highly debated. A 2012 study from Norway sought to determine whether diagnostic biopsy followed by a "wait and scan" approach or early resection would affect OS in patients with LGGs. The results showed a significantly better OS in the early resection group $(\mathrm{p}=0.01)$. The estimated 5-year survival was $60 \%$ for biopsy and watchful waiting versus $74 \%$ for early resection..$^{18}$ In 2014, Beiko et al. ${ }^{4}$ reported that IDHI status was independently associated with complete resection of enhancing disease $(93 \%$ complete resection in the mutant group vs $67 \%$ in the wild type, $\mathrm{p}<0.001$ ) in a series of 335 patients with malignant gliomas (either Grade III or Grade IV), suggesting that IDHI-mutant gliomas are more amenable to complete resection. IDHI status was also associated with survival difference between wild-type and mutant tumors. Complete resection of enhancing disease in patients with $I D H I$ wild-type tumors was associated with a median survival of 19.6 months versus 10.7 months for incomplete resection, thus supporting complete resection for Grade III and IV tumors. ${ }^{4}$ In this study, no survival benefit was observed with further resection of nonenhancing disease. In contrast, patients with an IDH1 mutation have an additional survival benefit with maximal resection of total tumor volume (median survival 9.75 years for $>5-\mathrm{ml}$ residual vs not reached for $<5 \mathrm{ml}$, $\mathrm{p}$ $=0.025) .{ }^{4}$ Although no similar study has been performed to examine LGGs, perhaps some inference can be made about the contrast-enhancing portion of tumors and accessibility based on $\mathrm{IDHI}$ status in planning and carrying out resection.

\section{Implications for Chemotherapy and Radiation}

There is evidence that the presence of $1 p 19 q$ codeletion is not only a positive prognostic indicator but also a strong predictor of chemosensitivity. In the Radiation Therapy Oncology Group (RTOG) 9402 trial, a prospective study of 291 patients with anaplastic oligodendroglioma and mixed anaplastic oligoastrocytomas, patients with tumors in which $1 p 19 q$ was codeleted had a 2-fold longer survival (14.7 vs 7.3 years median OS, $p=0.03$; and 8.4 vs 2.9 years median PFS, $p=0.001$ ) with the addition of procarbazine, lomustine, and vincristine (PCV) chemotherapy to radiation compared with radiation therapy alone. ${ }^{6}$ Even with radiation alone, the presence of $1 p 19 q$ codeletion was associated with longer OS (7.3 vs 2.7 years, $p<0.001$ ). For noncodeleted tumors, and for all patients combined, there was no survival difference in adding the chemotherapy to radiation. Interestingly, the Kaplan-Meier survival curves of codeleted and noncodeleted tumors did not diverge for 5 years, leaving us to wonder whether there are other factors complicating the role of $1 p 19 q$ codeletion in the chemosensitivity of these tumors. In the previously mentioned NOA-04 study, MGMT promoter methylation was also found to improve prognosis and render tumors more sensi- 
tive to radiation, which suggests the possibility that methylation inactivates genes responsible for radioresistance. ${ }^{39}$

The EORTC trial, ${ }^{36}$ which parallels RTOG 9402, also showed a significant advantage in OS (42.3 vs 30.6 months, $\mathrm{p}=0.018$ ) and PFS (24.3 months vs 13.2 months, $\mathrm{p}=0.0003$ ) to using upfront combination PCV chemotherapy and radiation in anaplastic oligodendroglial tumors (HR 0.75, 95\% CI 0.60-0.95). Although the difference in OS was borderline significant in the $1 p 19 q$ codeleted radiotherapy and PCV treatment group (HR 0.56, 95\% CI upper limit of $1.03, \mathrm{p}=0.0594)$, there was a much higher increase in PFS in codeleted tumors (106 months-from 50 months with radiotherapy alone to 156 months; HR $0.42,95 \%$ CI $0.24-0.74, \mathrm{p}=0.002$ ) with radiotherapy and PCV. In comparison, the PFS of noncodeleted tumors was 9 months in the radiotherapy-only group and 15 months in the radiotherapy and PCV group, an increase of only 6 months (HR 0.73, 95\% CI 0.56-0.97, p = 0.026). Although all $1 p 19 q$ codeleted tumors are $I D H$ mutated, not all $I D H$ mutated tumors are $1 p 19 q$ codeleted. The EORTC subset analysis, however, did demonstrate an independent predictive effect of $I D H$ mutation separate from $1 p 19 q$ codeletion, suggesting that either $1 p 19 q$ codeletion or IDH mutation is predictive of benefit from chemotherapy in anaplastic oligodendrogliomas. ${ }^{15}$ Together, the RTOG 9402 and EORTC studies reveal that the standard of care for oligodendroglial tumors that are $1 p 19 q$ codeleted should be combination chemotherapy and radiation.

Several open questions remain. First, given the biological similarities between IDH wild-type Grade III gliomas and GBMs, it is tempting to extrapolate that temozolomide should be beneficial in IDH wild-type GBMs, but neither RTOG 9402 nor EORTC 26951 saw a benefit to PCV in this subgroup. Second, although temozolomide is much more tolerable than PCV, it is not known whether it is as effective in Grade II and III gliomas. One retrospective study suggests that it is not, although it is more commonly used. ${ }^{23}$ Two other ongoing studies, the CODEL and CATNON trials, will probably answer the question of whether temozolomide can be used in combination with radiation to achieve similar effects to PCV, with less toxicity.

\section{Conclusions}

The preferred management of LGGs has been controversial, but recent advances are elucidating how molecular, genetic, and epigenetic alterations influence the pathogenicity of the different histological grades of gliomas. Better understanding of these mechanistic processes has led to a better understanding of LGGs that aids in diagnosis and planning for surgery, as well as promotion and creation of specific targeted therapies.

\section{Acknowledgment}

We thank Kristin Kraus, M.Sc., for editorial assistance in preparing this paper.

\section{References}

1. Ahmadi R, Dictus C, Hartmann C, Zürn O, Edler L, Hartmann M, et al: Long-term outcome and survival of surgically treated supratentorial low-grade glioma in adult patients.
Acta Neurochir (Wien) 151:1359-1365, 2009 [Erratum in Acta Neurochir (Wien) 151:1367, 2009]

2. Appin CL, Brat DJ: Molecular pathways in gliomagenesis and their relevance to neuropathologic diagnosis. Adv Anat Pathol 22:50-58, 2015

3. Balss J, Meyer J, Mueller W, Korshunov A, Hartmann C, von Deimling A: Analysis of the IDH1 codon 132 mutation in brain tumors. Acta Neuropathol 116:597-602, 2008

4. Beiko J, Suki D, Hess KR, Fox BD, Cheung V, Cabral M, et al: IDH1 mutant malignant astrocytomas are more amenable to surgical resection and have a survival benefit associated with maximal surgical resection. Neuro Oncol 16:81-91, 2014

5. Brennan CW, Verhaak RG, McKenna A, Campos B, Noushmehr H, Salama SR, et al: The somatic genomic landscape of glioblastoma. Cell 155:462-477, 2013

6. Cairncross G, Wang M, Shaw E, Jenkins R, Brachman D, Buckner J, et al: Phase III trial of chemoradiotherapy for anaplastic oligodendroglioma: long-term results of RTOG 9402. J Clin Oncol 31:337-343, 2013

7. Cavaliere R, Lopes MB, Schiff D: Low-grade gliomas: an update on pathology and therapy. Lancet Neurol 4:760-770, 2005

8. Chaichana KL, McGirt MJ, Laterra J, Olivi A, QuiñonesHinojosa A: Recurrence and malignant degeneration after resection of adult hemispheric low-grade gliomas. J Neurosurg 112:10-17, 2010

9. Choi C, Ganji SK, DeBerardinis RJ, Hatanpaa KJ, Rakheja $\mathrm{D}$, Kovacs Z, et al: 2-hydroxyglutarate detection by magnetic resonance spectroscopy in IDH-mutated patients with gliomas. Nat Med 18:624-629, 2012

10. Christensen BC, Smith AA, Zheng S, Koestler DC, Houseman EA, Marsit CJ, et al: DNA methylation, isocitrate dehydrogenase mutation, and survival in glioma. J Natl Cancer Inst 103:143-153, 2011

11. Cohen AL, Holmen SL, Colman H: IDH1 and IDH2 mutations in gliomas. Curr Neurol Neurosci Rep 13:345, 2013

12. Dang L, White DW, Gross S, Bennett BD, Bittinger MA, Driggers EM, et al: Cancer-associated IDH1 mutations produce 2-hydroxyglutarate. Nature 462:739-744, 2009

13. Dunn GP, Andronesi OC, Cahill DP: From genomics to the clinic: biological and translational insights of mutant IDH1/2 in glioma. Neurosurg Focus 34(2):E2, 2013

14. Elkhaled A, Jalbert LE, Phillips JJ, Yoshihara HA, Parvataneni R, Srinivasan R, et al: Magnetic resonance of 2-hydroxyglutarate in IDH1-mutated low-grade gliomas. Sci Transl Med 4:116ra115, 2012

15. Erdem-Eraslan L, Gravendeel LA, de Rooi J, Eilers PH, Idbaih A, Spliet WG, et al: Intrinsic molecular subtypes of glioma are prognostic and predict benefit from adjuvant procarbazine, lomustine, and vincristine chemotherapy in combination with other prognostic factors in anaplastic oligodendroglial brain tumors: a report from EORTC study 26951. J Clin Oncol 31:328-336, 2013

16. Figueroa ME, Abdel-Wahab O, Lu C, Ward PS, Patel J, Shih A, et al: Leukemic IDH1 and IDH2 mutations result in a hypermethylation phenotype, disrupt TET2 function, and impair hematopoietic differentiation. Cancer Cell 18:553-567, 2010

17. Hegi ME, Diserens AC, Gorlia T, Hamou MF, de Tribolet N, Weller M, et al: MGMT gene silencing and benefit from temozolomide in glioblastoma. N Engl J Med 352:997-1003, 2005

18. Jakola AS, Myrmel KS, Kloster R, Torp SH, Lindal S, Unsgård G, et al: Comparison of a strategy favoring early surgical resection vs a strategy favoring watchful waiting in lowgrade gliomas. JAMA 308:1881-1888, 2012

19. Keevil SF, Barbiroli B, Brooks JC, Cady EB, Canese R, Carlier P, et al: Absolute metabolite quantification by in vivo 
NMR spectroscopy: II. A multicentre trial of protocols for in vivo localised proton studies of human brain. Magn Reson Imaging 16:1093-1106, 1998

20. Keles GE, Lamborn KR, Berger MS: Low-grade hemispheric gliomas in adults: a critical review of extent of resection as a factor influencing outcome. J Neurosurg 95:735-745, 2001

21. Kim BY, Jiang W, Beiko J, Prabhu SS, DeMonte F, Gilbert MR, et al: Diagnostic discrepancies in malignant astrocytoma due to limited small pathological tumor sample can be overcome by IDH1 testing. J Neurooncol 118:405-412, 2014

22. Lang FF, Gilbert MR: Diffusely infiltrative low-grade gliomas in adults. J Clin Oncol 24:1236-1245, 2006

23. Lassman AB, Iwamoto FM, Cloughesy TF, Aldape KD, Rivera AL, Eichler AF, et al: International retrospective study of over 1000 adults with anaplastic oligodendroglial tumors. Neuro Oncol 13:649-659, 2011

24. Leu S, von Felten S, Frank S, Vassella E, Vajtai I, Taylor E, et al: IDH/MGMT-driven molecular classification of low-grade glioma is a strong predictor for long-term survival. Neuro Oncol 15:469-479, 2013

25. McGirt MJ, Chaichana KL, Attenello FJ, Weingart JD, Than $\mathrm{K}$, Burger PC, et al: Extent of surgical resection is independently associated with survival in patients with hemispheric infiltrating low-grade gliomas. Neurosurgery 63:700-708, 2008

26. Phillips JJ, Aranda D, Ellison DW, Judkins AR, Croul SE, Brat DJ, et al: PDGFRA amplification is common in pediatric and adult high-grade astrocytomas and identifies a poor prognostic group in IDH1 mutant glioblastoma. Brain Pathol 23:565-573, 2013

27. Pope WB, Prins RM, Thomas MA, Nagarajan R, Yen KE, Bittinger MA, et al: Non-invasive detection of 2-hydroxyglutarate and other metabolites in IDH1 mutant glioma patients using magnetic resonance spectroscopy. J Neurooncol 107:197-205, 2012

28. Recht LD, Lew R, Smith TW: Suspected low-grade glioma: is deferring treatment safe? Ann Neurol 31:431-436, 1992

29. Reijneveld JC, Sitskoorn MM, Klein M, Nuyen J, Taphoorn MJ: Cognitive status and quality of life in patients with suspected versus proven low-grade gliomas. Neurology 56:618623, 2001

30. Shaw EG, Berkey B, Coons SW, Bullard D, Brachman D, Buckner JC, et al: Recurrence following neurosurgeon-determined gross-total resection of adult supratentorial low-grade glioma: results of a prospective clinical trial. J Neurosurg 109:835-841, 2008

31. Smith JS, Chang EF, Lamborn KR, Chang SM, Prados MD, Cha S, et al: Role of extent of resection in the long-term outcome of low-grade hemispheric gliomas. J Clin Oncol 26:1338-1345, 2008
32. Sturm D, Witt H, Hovestadt V, Khuong-Quang DA, Jones DT, Konermann C, et al: Hotspot mutations in H3F3A and IDH1 define distinct epigenetic and biological subgroups of glioblastoma. Cancer Cell 22:425-437, 2012

33. Sun H, Yin L, Li S, Han S, Song G, Liu N, et al: Prognostic significance of IDH mutation in adult low-grade gliomas: a meta-analysis. J Neurooncol 113:277-284, 2013

34. Taylor JW, Chi AS, Cahill DP: Tailored therapy in diffuse gliomas: using molecular classifiers to optimize clinical management. Oncology (Williston Park) 27:504-514, 2013

35. Turkalp Z, Karamchandani J, Das S: IDH mutation in glioma: new insights and promises for the future. JAMA Neurol 71:1319-1325, 2014

36. van den Bent MJ, Brandes AA, Taphoorn MJ, Kros JM, Kouwenhoven MC, Delattre JY, et al: Adjuvant procarbazine, lomustine, and vincristine chemotherapy in newly diagnosed anaplastic oligodendroglioma: long-term follow-up of EORTC brain tumor group study 26951. J Clin Oncol 31:344-350, 2013

37. Wakimoto H, Tanaka S, Curry WT, Loebel F, Zhao D, Tateishi K, et al: Targetable signaling pathway mutations are associated with malignant phenotype in IDH-mutant gliomas. Clin Cancer Res 20:2898-2909, 2014

38. Wessels PH, Weber WE, Raven G, Ramaekers FC, Hopman AH, Twijnstra A: Supratentorial grade II astrocytoma: biological features and clinical course. Lancet Neurol 2:395403, 2003

39. Wick W, Hartmann C, Engel C, Stoffels M, Felsberg J, Stockhammer F, et al: NOA-04 randomized phase III trial of sequential radiochemotherapy of anaplastic glioma with procarbazine, lomustine, and vincristine or temozolomide. J Clin Oncol 27:5874-5880, 2009

40. Yan H, Parsons DW, Jin G, McLendon R, Rasheed BA, Yuan W, et al: IDH1 and IDH2 mutations in gliomas. N Engl J Med 360:765-773, 2009

\section{Author Contributions}

Conception and design: Ravindra, Colman, Chen. Acquisition of data: Salzman, Prescot, Colman. Analysis and interpretation of data: Salzman, Prescot. Drafting the article: Chen, Ravindra. Critically revising the article: all authors. Reviewed submitted version of manuscript: all authors. Approved the final version of the manuscript on behalf of all authors: Chen. Administrative/ technical/material support: Colman. Study supervision: Colman.

\section{Correspondence}

Ricky Chen, Department of Neurology, Clinical Neurosciences Center, University of Utah, 175 N. Medical Dr. E, Salt Lake City, UT 84132.email: ricky.chen@hsc.utah.edu. 